\title{
Réflexion sur le développement alternatif à la culture de la coca en Amérique andine
}

Oléagineux, Corps Gras, Lipides. Volume 8, Numéro 6, 621-5, Novembre - Décembre 2001, Dossier : L'avenir des cultures pérennes

Auteur(s) : Jean-Claude FOLLIN, Franck RIVANO, Cirad-Cp, TA 80/PS3, boulevard de la Lironde, 37398 Montpellier Cedex 5.

Résumé : Dans les années 1965-1975, on observe un développement très important des cultures illicites et de la consommation de drogues, qui s'accentue encore dans la décennie suivante. Ce développement est concomitant à deux phénomènes mondiaux indépendants : d'une part, l'ouverture des agricultures du Sud à l'économie de marché et, d'autre part, des changements culturels profonds dans les pays du Nord, avec les premières manifestations de dérégulation sociale. Les premières actions internationales, dans le domaine de la réduction de l'offre, pour le contrôle de la culture des plantes productrices de drogues ont commencé au milieu des années 70 en Asie sur la culture du pavot (au travers du Pnucid - programme des Nations unies pour le contrôle international des drogues) ; elles se sont ensuite développées en Amérique latine sur la culture de la coca (création de la Comisión interamericana para el control del abuso de drogas - Cicad, en 1986), puis dans le bassin méditerranéen au début des années 90 sur la culture du chanvre (programmes UE/MED). Les premières années, cette lutte s'est opérée sous le concept de cultures de substitution (ou cultures alternatives) : les parcelles de culture illicites étaient arrachées et remplacées par d'autres productions. L'analyse des échecs répétés de ce type d'intervention a montré que les régions productrices étaient presque toujours des régions marginalisées d'un point de vue socio-économique et que la lutte contre les cultures illicites n'était pas uniquement une affaire de développement de nouvelles cultures. Il était, en fait, nécessaire de créer les conditions d'une nouvelle économie agricole. Ainsi, le concept de développement alternatif a peu à peu émergé pour aboutir aux grands principes de base énoncés, en 33 points, lors de l'assemblée générale des Nations unies du 8 septembre 19981.

Summary : The cultivation of coca (Erythroxylum coca Lam.), a perennial shrub of the order Geraniales, goes back to ancient times in Bolivia and Peru, but its extension over wide areas is quite recent, beginning around 1960, with a period of strong expansion between 1975 and 1985 (coca boom). In 1992, it then started to decline in Bolivia and Peru, but continued to progress in Colombia, with an intensification of the crops. The integrated (alternative) development policies implemented over the last decade have had positive consequences for infrastructures (roads, public health facilities, education, etc.), but have had little success in replacing illicit crops with legal cash crops, primarily for market and price reasons. As a result, the forced eradication operations carried out to make up for the deficit have created serious social conflicts. The socio-economic situations in regions affected by illicit crops, and those in regions with production potential, may be very different depending on the country and the continent. Nevertheless, controlling illicit crops needs to be conceived in terms other than repression, and agricultural development needs to be proposed in terms other than prices and competitiveness. That means introducing fairer policies and markets, so that farmers are not marginalized and do not turn to illegal production.

Keywords : illicit crops, coca plant, alternative development. 


\section{ARTICLE}

\section{La culture de la coca en Amérique andine}

La culture de la coca (Erythroxylum coca Lam. et E. novogranatense (Morris) Hieron, arbustes pérennes de la famille des géraniales) est très ancienne en Bolivie et au Pérou : les archéologues la font remonter à 4500 ans et les peuples de la zone Inca la consommaient comme stimulant ainsi que pour des usages médicaux et divinatoires. La colonisation espagnole tenta de l'interdire sauf dans les régions minières pour lesquelles elle installa dans la province de La Paz (Yungas) une production qui donna lieu à des échanges commerciaux importants. Il existe de nombreuses variétés adaptées à différents écosystèmes, mais la grande culture se situe essentiellement dans les zones tropicales humides où elle entre en concurrence avec les cultures pérennes habituelles de ces régions : café, cacao, hévéa, fruitiers, etc.

L'extension des cultures est assez récente et débute vers la fin des années 60 , avec une période de forte expansion dans la décennie 1975-1985 de " boom de la coca ", expansion due principalement à une forte demande des pays du Nord, un contexte économique hyperinflationniste et l'existence de républiques « cocaineras ». En 1986, il est estimé qu'en Bolivie la coca occupe entre 53,6 et $64,6 \%$ du PIB [1].

Ensuite, à partir de 1992, on observe un déclin en Bolivie et au Pérou lié à diverses raisons : baisse des prix dans les pays consommateurs du Nord, répression efficace dans les circuits de trafic de la cocaïnebase entre la Bolivie et le Pérou d'une part, la Colombie d'autre part, établissement de politiques antidrogue des pays concernés, etc.

Actuellement, la situation est très contrastée suivant les pays : au Pérou, il est difficile d'avoir une idée globale précise car la culture est très diffuse et concerne 25 départements et 16 vallées, souvent peu accessibles, mais il semble qu'après une baisse estimée de 140000 à 40000 ha de 1994 à 1999 (selon les chiffres officiels, probablement trop optimistes), la culture redémarre, parallèlement à une augmentation du prix des feuilles sèches achetées aux paysans. À l'inverse, en Colombie, la culture n'a jamais régressé et elle est passée de 45000 ha en 1994 à 122000 ha en 2000. Dans ce pays, il semble que la baisse des prix ait été compensée par une intensification des cultures et par le développement de grandes plantations conduites de façon moderne (voir tableau).

En Bolivie, la situation est mieux connue car les zones de production sont limitées à deux régions, et il apparaît que l'éradication commencée dans Chapare de manière persuasive, avec une prime à l'arrachage, et poursuivie de manière plus brutale, a été efficace : la culture de la coca y est devenue anecdotique. L'éradication forcée est désormais envisagée dans les Yungas pour les surfaces excédentaires. II ne resterait donc qu'environ 10000 ha autorisés dans la zone de culture traditionnelle.

Ce succès incontestable dans le Chapare a toutefois un coût social insupportable car il laisse 40000 familles sans ressources, dont un premier indicateur, facile à mesurer, est une déscolarisation importante des enfants. Il est à prévoir que les paysans, désespérés par une misère qu'ils savent désormais ne pas venir de la fatalité, renoueront avec les émeutes du type de celles de septembre 2000. Il est à craindre que la production ne se déplace vers des zones moins accessibles et qu'une situation de type colombien ne s'installe. 
Le Chapare est aussi un lieu exemplaire d'observation car des sommes très importantes y ont été allouées pour le développement alternatif aux cultures de coca, dans un premier temps par l'Usaid, et plus récemment par l'UE. Les stratèges de cette nouvelle forme de développement intégré ont ainsi eu le temps et les moyens de définir des projets et de les mettre en œuvre. Un premier bilan peut donc être établi. De l'avis de tous, il est pour le moins contrasté : s'il est incontestablement positif pour les infrastructures (moyens de communications, édifices sociaux, aide aux municipalités, etc.), il est en revanche tout à fait négatif pour les tentatives de développement de productions alternatives à la coca amenant un revenu décent. La raison peut apparaître simple : aucune des spéculations agricoles proposées ne trouve de marchés, à l'exception toutefois de la banane pour laquelle il existe un marché limité en Argentine mais qui est une culture exigeante en connaissances techniques et en intrants, et qui est donc hors de portée de la grande majorité des paysans du Chapare. Cependant, avant d'arriver à cette conclusion ultime, il n'est pas inutile de réfléchir sur les ambiguïtés d'un développement alternatif qui prône l'intégration des actions, mais qui se retrouve inopérant sur le problème de la production agricole, de la même manière que les projets de développement de cultures de substitution du début des années 80 .

\section{Le concept de développement alternatif}

Pour l'essentiel, le développement alternatif est un ensemble d'activités intégrées (économiques, sociales, politiques, etc.) réalisées par l'État, la société civile et la coopération internationale, orientées pour produire des revenus, construire des infrastructures, et créer un nouveau contexte socioéconomique et culturel, afin d'améliorer les niveaux de développement des populations des zones concernées par les cultures illicites et leur transformation.

L'application sur le terrain a montré que, pour l'Amérique latine, sept points étaient particulièrement importants [2].

\section{* Intégration et la diversification des moyens}

Le développement alternatif est un ensemble de politiques et d'actions multi-sectorielles et pluridisciplinaires orienté vers la substitution d'une économie de monoculture par une économie de cultures diversifiées et d'activités économiques durables et respectueuses de l'environnement. Le développement alternatif ne se limite pas à changer de culture mais doit générer une nouvelle économie " alternative ", ce qui implique une diversification des actions productives.

\section{* Volonté des acteurs}

La communauté nationale, en particulier dans les zones touchées, doit être convaincue du bien-fondé des actions à engager. Au niveau de la production, il est aussi indispensable d'avoir l'accord de la communauté paysanne. II est ainsi préférable de commencer les plans de développement avec les paysans non producteurs de drogues ou avec ceux qui désirent abandonner la culture de la coca. On peut ainsi espérer un effet d'entraînement. 


\section{* Participation des acteurs}

Les paysans et leurs organisations doivent participer à l'élaboration des plans et à leur exécution ainsi qu'à leur évaluation. Il faut partir des organisations sociales existantes, et non en créer ex nihilo, et prendre en compte les savoirs locaux dans le transfert des innovations.

\section{* Compétitivité sur le marché}

Dans l'élaboration des projets, il est indispensable de prendre en compte des produits ou des activités qui correspondent à la demande - par ordre d'importance - du marché local, national, régional ou international. II faut produire des biens vendables qui contribuent à améliorer le niveau économique des populations concernées.

\section{* Volonté politique}

II n'existe pas de gouvernement qui affiche un franc désintérêt pour les problèmes posés par la production ou la consommation de drogues. Il est, en revanche, plus rare de voir énoncer et appliquer des plans cohérents de lutte, avec une stratégie, un ensemble de lois adéquates, des institutions motivées, des relations de coopération régionale et internationale et des engagements financiers conséquents.

\section{* Interdiction et éradication des cultures illicites}

Compte tenu de l'expérience des pays andins, il est clair que les méthodes coercitives (notamment l'éradication forcée) ne peuvent être un préalable ni être concomitantes aux plans de développement alternatif. Le développement doit créer les conditions qui permettent la régression des cultures illicites par des voies négociées.

\section{* Problèmes de sécurité}

Ils sont souvent liés au narcotrafic, mais les autorités ne doivent pas confondre les petits paysans producteurs et les trafiquants. II est indispensable que les États réaffirment leur présence dans les zones productrices de coca, mais ces derniers doivent orienter les actions d'interdiction essentiellement vers les trafiquants, faute de quoi la confiance réciproque nécessaire à la réussite des plans de développement ne s'établira pas.

\section{* Coopération régionale et internationale}

S'il existe des différences importantes dans les conditions de production des cultures illicites dans les pays andins, il est cependant possible de trouver un terrain d'entente pour la création d'un espace commun d'action " pays andins - pays consommateurs du Nord ". II est difficile d'imaginer que la lutte contre la drogue puisse trouver une solution indépendante dans chacun des pays concernés, que ce soit pour la production, le trafic ou la consommation.

\section{Difficultés du développement alternatif}

\section{Les ambiguïtés}

L'application du développement alternatif, tel qu'il est conçu actuellement, soulève trois types de problèmes : des problèmes d'éthique, des problème politiques et des problèmes économiques [3]. 


\section{* Le problème éthique}

Parler de problème éthique sous-entend souvent problème intellectuel, sinon d'intellectuels ; or il s'agit d'un aspect sur lequel le visiteur européen est le plus souvent interpellé par les habitants des zones productrices de coca et qui se traduit par la question : "Pourquoi nous demandez-vous de résoudre vos problèmes qui relèvent de votre culture et non de la nôtre ? " Une autre remarque fréquente concerne l'incapacité des pays du Nord à contrôler l'exportation des produits (précurseurs) nécessaires à la fabrication de la pâte de cocaïne. Cela revient à poser le problème du statut de la coca qui n'est pas une culture illicite pour les paysans andins (excepté en Colombie), de l'utilisation de la feuille coca (dont les effets nocifs n'ont jamais été démontrés, dont la vente est autorisée sous forme de sachets destinés à faire des infusions et qui entre dans la composition de certaines boissons très populaires), et du statut du planteur de coca, qui n'est pas un producteur de cocaïne, ni un délinquant (en tout cas, il ne se considère pas comme tel). En outre, le paysan cocaïculteur, en Bolivie et au Pérou, appartient le plus souvent à une association ou à une fédération officielle de producteurs et peut vendre sa production de feuilles à des organismes agréés dans les zones autorisées.

En d'autres termes, le développement alternatif apparaît aux paysans comme un instrument au service d'une cause qui ne les concerne pas.

\section{* Le problème politique}

En l'absence de mesures particulières, l'alternative laissée au paysan est d'abandonner une culture traditionnelle, bien rémunérée, aux débouchés assurés, pour une autre production aux prix incertains, sur un marché libre aux tendances baissières (café, ananas, palmito, etc.) qui, dans le meilleur des cas n'amènera un revenu que plusieurs années après. À cela il faut ajouter que les autorités politiques ${ }^{2}$ demandent que les aides (en particulier le crédit) soient liées à l'abandon immédiat de la culture de la coca, à l'échelon individuel, ou plus fort encore, à l'échelle de la zone qui doit être "libre de coca ». Cette exigence identifie encore plus le développement alternatif à la répression. Elle est de toute façon inopérante car l'agriculteur, quelle que soit sa situation, gère des risques (le plus souvent climatiques et économiques) et met rarement "tous ses œufs dans le même panier ". Dans le cas présent, l'agriculteur ne peut accepter cette conditionnalité, pour des raisons économiques évidentes.

Dans ces conditions, on comprend aisément que le paysan (la communauté) sorte de la légalité et tente d'assurer sa survie économique en traitant avec le marché illégal pour la vente, puis plus en aval, avec les transformateurs. Cela pose des problèmes politiques très sérieux dans la mesure où il s'agit d'un premier pas vers la dissidence.

Par ailleurs, l'implication des États-Unis dans l'éradication forcée et l'image parfois brouillée de la politique de l'Union européenne accrédite l'idée que les gouvernements locaux ne sont plus que les relais de décisions prises " ailleurs ». Cette image - vraie ou fausse - est une composante importante de l'idéologie de nouveaux mouvements révolutionnaires, comme par exemple celui des zapatistes au Mexique, où la revendication anti-mondialiste est très forte.

\section{* Les problèmes économiques}

Les projets sont conçus au niveau national pour être appliqués à un niveau provincial. Tout se passe comme si la zone concernée était isolée du reste du pays. Donner des avantages trop importants (avantages fiscaux ou subventions aux entreprises, constructions d'infrastructures, etc.) crée à terme des distorsions et des situations d'iniquité difficilement gérables. De même, à l'échelle régionale, 
l'expérience acquise démontre qu'en attaquant le problème dans un seul pays, on provoque un déplacement de ce problème vers les pays limitrophes ${ }^{3}$. La logique voudrait donc que les aides soient coordonnées, notamment dans le cas des projets de développement ruraux, afin que les productions de nouvelles denrées soient complémentaires plutôt que concurrentes lorsqu'il s'agit de produits destinés au marché international.

En fait, il apparaît que les projets de développement alternatif ont apporté des améliorations appréciables, en particulier dans les infrastructures - dont on peut espérer qu'elles auront à terme un effet positif sur le développement agricole en favorisant l'accès des exploitations aux marchés - mais que pour rendre ces projets viables en assurant une production agricole durable, il est nécessaire de prendre en considération des facteurs qui dépassent le cadre régional restreint du projet lui-même.

Les difficultés de terrain

Les grands principes du développement alternatif (tels qu'énoncés plus haut) s'adressent essentiellement à la production familiale (qui représente la totalité de la production en Bolivie et au Pérou et la grande majorité en Colombie), et sont en théorie parfaitement applicables. Les producteurs appartiennent aux classes les plus défavorisées de la société, qui subissent une quadruple précarité : citoyenne (éloignement, voire absence des services publics de base), foncière (absence fréquente de titre de propriété), économique (prédominance de l'autosubsistance) et sanitaire (une mortalité infantile élevée). Leur aspiration n'est pas de s'enrichir avec la coca mais de vivre décemment et qu'il soit mis fin aux violences dont ils sont les principales victimes. En outre, de leur origine culturelle, ils ont conservé des structures de vie et d'aide communautaires ${ }^{4}$ qui permettent une mobilisation facilitée des organisations villageoises, pour peu toutefois que la confiance soit établie, ce qui représente vraisemblablement, dans le contexte actuel, la tâche la plus ardue.

Dans la réalité, ces principes sont difficiles à mettre en œuvre, d'une part, à cause des rapports de force politiques qui empêchent le développement des actions les plus sensibles (contrôle des trafiquants, réformes foncières, organisation du crédit, etc.) et, d'autre part, parce que le développement alternatif se heurte à deux logiques opposées :

1. Les principes du développement alternatif peuvent s'appliquer, il n'y a pas de conditions d'éradication, mais aucune culture ne pouvant concurrencer la coca - soit parce que les denrées ne sont pas adaptées au marché intérieur ou ne sont pas compétitives sur le marché international, soit parce que les cultures "prétendantes » nécessitent des conditions agro-écologiques ou socioéconomiques qui excluent, de fait, une proportion élevée des producteurs actuels ${ }^{5}$ - les paysans reçoivent les aides, et continuent à cultiver la coca qui est la seule culture leur assurant un revenu stable.

2. La coercition pallie les insuffisances de la substitution, l'éradication forcée est menée sur une grande échelle, il y a des succès initiaux dans la régression des cultures mais faute d'alternatives crédibles, la répression sélectionne les paysans les plus aptes à entrer dans l'illégalité. Les prix des feuilles augmentent, rendant les alternatives encore moins crédibles (ils ont été multipliés par 5 en 2000 en Bolivie, par 3 au Pérou). L'insécurité s'installe et les cultures de coca deviennent cette fois vraiment illicites (situation colombienne).

Dans les deux cas il y a échec, car on reste dans une conception productiviste de l'agriculture et dans une logique de substitution, avec l'impossibilité de trouver une culture dont les prix des produits 
seraient alignés sur le marché international, et qui donnerait des revenus, sinon équivalents à ceux de la coca, tout au moins suffisants pour assurer les besoins essentiels ${ }^{6}$ des familles en matière d'alimentation, de santé et de scolarisation des enfants.

Notes :

${ }^{1}$ Measures to enhance international cooperation to counter the world drug problem - E. Action plan on international cooperation on the eradication of illicit drug crops and on alternative development, pp. 20-24, site web de l'UNDCP (www.undcp.org).

${ }^{2}$ Et certains pays comme les États-Unis. En principe, même si l'accord sur ce point n'est pas absolu, I'UE ne retient pas cette conditionnalité et, en conséquence, rencontre des difficultés dans l'exécution de ses projets.

${ }^{3}$ Point 16 de la résolution du Parlement européen sur le plan Colombie ( $1^{\mathrm{er}}$ février 2001).

${ }^{4}$ Même lorsqu'il émigre des zones d'altitude vers les zones tropicales humides, le paysan des Andes conserve des liens forts avec la communauté d'origine et reprend, sur une échelle géographique réduite, le système Inca de cultures par étages écologiques qui permettait de limiter les risques climatiques [4].

${ }^{5}$ La culture de la coca a un triple avantage : c'est une culture traditionnelle, elle procure un revenu assuré, réparti sur toute l'année (3 à 4 récoltes par an).

${ }^{6}$ Pendant les événements de septembre 2000, les producteurs de coca du Chapare demandaient l'autorisation de cultiver un quart d'hectare de coca, ce qui correspond à un revenu brut de l'ordre de 400 à 500 dollars US (pour un prix de 1dollar le kilo de feuilles).

${ }^{7}$ En Colombie, dans les provinces du Putumayo, Caqueta, Bolivar et Norte Santander, 40000 familles ont certes signé un "pacte social de développement alternatif ", mais dans des conditions de guerre civile telles qu'on peut s'interroger sur le choix qui leur est laissé.

${ }^{8}$ On peut imaginer par exemple des systèmes de mise en place d'une arboriculture (hévéas, fruitiers, etc.) dans des plantations de coca qui disparaîtront sous l'ombrage après 3 à 4 années de croissance.

${ }^{9}$ En Colombie, les « pactes sociaux de développement alternatif » autorisent l'éradication progressive des cultures illicites dans un laps de temps déterminé, généralement un an après la signature du pacte, ce qui va dans la bonne direction mais reste toutefois insuffisant pour l'établissement d'une culture pérenne de remplacement, sauf à prévoir, en association, des cultures à mise en production rapide.

${ }^{10} \mathrm{Si}$ tous les spécialistes de la lutte antidrogue s'accordent pour penser que les régions productrices sont toujours parmi les plus pauvres, ils ne perçoivent pas toujours la dynamique d'appauvrissement et de marginalisation, notamment foncière et citoyenne, qui touche certaines régions, les plaçant ainsi dans les zones « candidates".

\section{CONCLUSION}

Les difficultés actuelles, en Amérique latine, du développement alternatif (principalement dans sa composante agricole) doivent aussi être observées à partir de comparaisons avec d'autres régions du monde potentiellement touchées par le même problème. 
En Bolivie et au Pérou, l'extension de la culture de la coca à partir du début des années 70 a été concomitante au phénomène mondial de la monétarisation de l'agriculture des pays du Sud et au déclin du latifundisme (réforme agraire de 1953 en Bolivie, un peu plus tard au Pérou). La cocaïculture a été pour de nombreux paysans la source initiale de revenus monétaires. II en va de même de la Colombie, mais dans des conditions différentes, dans la mesure où la coca n'est pas une culture traditionnelle mais une culture d'introduction récente, très liée à la guerre civile.

En Afrique de l'Ouest, dans les cas étudiés par Léonard [5], la situation est très différente. II n'y avait pas de problèmes fonciers dans cette même période et le développement agricole s'est fait sur la base de cultures de rente d'exportation : cultures pérennes au Sud, coton au Nord. Les cultures illicites, essentiellement les cultures de cannabis, viennent plus tard, en concurrence (ou en complément) d'autres cultures déjà bien établies. La situation est donc a priori plus favorable pour les cultures autorisées.

Toutefois, dans les deux cas, on constate que dans les raisons fondamentales de l'établissement ou du maintien des cultures illicites, il y a le même problème du prix des denrées agricoles et que l'approche de la production agricole sous la forme " offre/demande » régie par les règles du marché international libéral conduit à une impasse. En Amérique latine, aucune proposition ne rencontre l'adhésion volontaire des agriculteurs ${ }^{7}$ et, en Afrique de l'Ouest, les cultures de rente traditionnelles (notamment le café et le cacao) ne donnant plus de revenus satisfaisants, certaines zones entrent dans l'engrenage des cultures interdites. En définitive, le problème des cultures illicites rejoint le problème général de la rémunération des produits agricoles et des agriculteurs.

Pour la substitution des cultures illicites, il y a lieu toutefois de distinguer deux phases successives : la période de transition et le maintien de la nouvelle culture de rente. Dans la première phase, une logique normale de développement agricole voudrait que l'agriculteur n'abandonne que progressivement la culture illicite et que des crédits lui permettent d'établir une nouvelle culture ${ }^{8}$. C'est malheureusement rarement le cas $^{9}$ et la conditionnalité du crédit liée à l'arrachage préalable de la coca bloque souvent les projets de développement alternatif dès les premiers stades. Cette exigence conduit toujours, comme cela a été décrit plus haut, à des rapports de force entre les autorités et les communautés paysannes.

La seconde phase concerne le maintien de la culture de substitution et les politiques à mettre en place pour assurer des prix compétitifs. Une des politiques extrêmes consiste à proposer la libéralisation totale des drogues dans les pays du Nord, ce qui aboutirait certainement à une baisse des prix et à une régulation des productions au Sud mais ne résoudrait en rien la situation de pauvreté des paysanneries dont la préoccupation est plutôt la hausse des prix des cultures licites que la baisse des prix des produits stupéfiants.

L'autre alternative est donc de revoir les concepts de l'aide au développement afin de dépasser le modèle productiviste en contribuant à faire émerger des politiques et des marchés plus équitables évitant aux paysanneries d'être marginalisées ${ }^{10}$ par la mondialisation. Une approche multifonctionnelle de l'agriculture qui prendrait en compte les externalités négatives et coûteuses des drogues dans les pays du Nord (santé, délinquance, etc.) et du Sud (perversion des réseaux commerciaux traditionnels, sécurité, pollution de l'environnement par les produits d'extraction, etc.) pourrait contribuer à cette réflexion. 


\section{REFERENCES}

1. DORY D, ROUX JC (1998). De la coca à la cocaïne : un itinéraire bolivien. In : Drogue et reproduction sociale dans le tiers monde, coll. " autrepart », Bondy : éd. de l'aube/Orstom : 21-46.

2. CABIESES H, MUSSO E (1999). Desarrollo alternativo y desarrollo rural. Debate sobre sus limitades y posibilidades. Lima : IICA ed. ; 299 pages.

3. LASERNA R (1999). Las etapas del desarrollo alternativo en Bolivia. In : Desarrollo alternativo y desarrollo rural. Debate sobre sus limitades y posibilidades. Lima : IICA ed. : 149-55.

4. FRANQUEVILLE A (2000). La Bolivie, d'un pillage à l'autre. Toulouse : Éditions de I'IRD/Presses universitaires du Mirail, Université de Toulouse-Le Mirail ; 292 p.

5. LÉONARD E (1998). Crise des économies de plantations en Afrique de l'Ouest : les cas ivoirien et ghanéen. In : Drogue et reproduction sociale dans le tiers monde, coll. " autrepart ". Bondy : éd. de l'aube/Orstom : 79-99. 
Tableau. Caractéristiques de la production de Coca en Bolivie, au Pérou et en Colombie.

\begin{tabular}{|c|c|c|c|}
\hline 1. Cultures et production & Bolivie & Pèrou & Colombie \\
\hline Cultures (ha) sabn les types et les zones (1998) & $\begin{array}{l}55000 \text { (1994) } \\
1994: 48000 \\
1997: 45800 \\
1998: 38800 \\
2000:: 20000 \\
\text { C. legals (Yungs) : } 12500 \\
\text { C. illicites (Chapare, Yungas) : } 26000\end{array}$ & \multirow{6}{*}{ 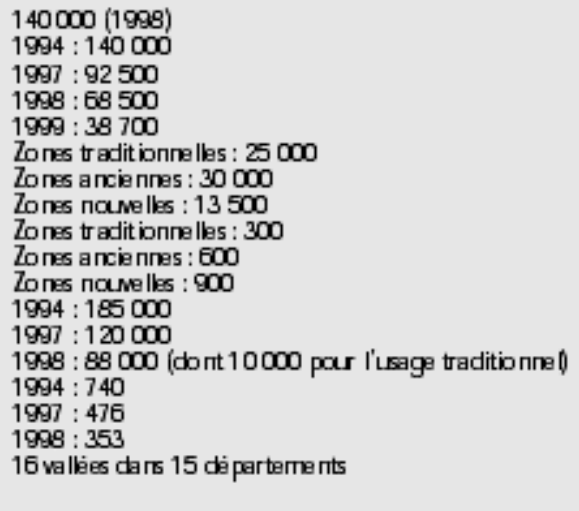 } & $\begin{array}{l}110000(1998)(1) \\
1994: 46500 \\
1997: 79500 \\
1998: 102000 \\
2000: 122000 \\
\text { Grandes exp bitatiors : } 54000 \\
\text { Exploitatiors farmilia les : } 47000\end{array}$ \\
\hline Rendements moyens (kg/ha de feuilles seches) & $\begin{array}{l}\text { Los Yungas : } 900 \\
\text { Chapare : } 2600\end{array}$ & & $\begin{array}{l}\text { Grandes exploitatiors : } 3600 \\
\text { Exploitatiors farniliales : } 1600\end{array}$ \\
\hline Production de feuilles (estirnation, tonnes) & $\begin{array}{l}1994: 90000 \\
1997: 70000\end{array}$ & & $\begin{array}{l}1994: 125000 \\
1997: 213000\end{array}$ \\
\hline Production potentielle de cocaine & $\begin{array}{l}1998: 55000 \text { (do nt } 8000 \text { par l'usage traditionne ) } \\
1994: 3599\end{array}$ & & $\begin{array}{l}1998: 272000 \text { (dont } 500 \text { par l'usage traditionnel) } \\
1994: 498\end{array}$ \\
\hline & $1997: 280$ & & $1997: 852$ \\
\hline $\begin{array}{l}\text { (HCHestimation, tonnes) } \\
\text { Zones de production }\end{array}$ & $\begin{array}{l}1998: 156 \\
\text { Los Yungas de La Paz } \\
\text { Tró pion de Cochabamba et Apob }\end{array}$ & & $\begin{array}{l}1998: 1087 \\
\text { Production dis perseie ders } 25 \text { des } 33 \text { dejparternents }\end{array}$ \\
\hline 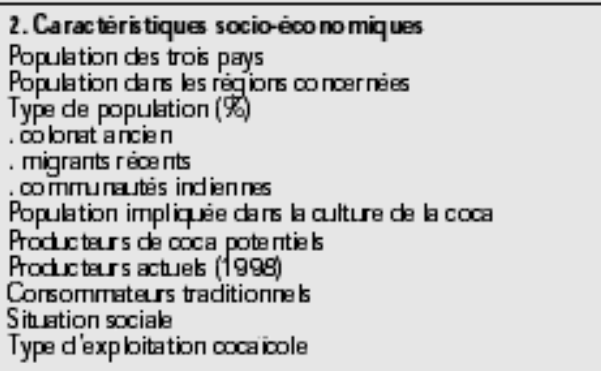 & 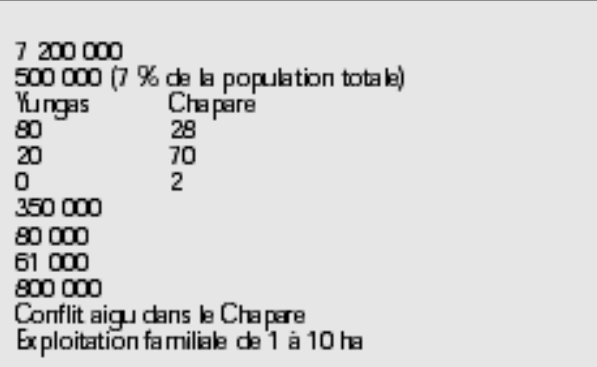 & 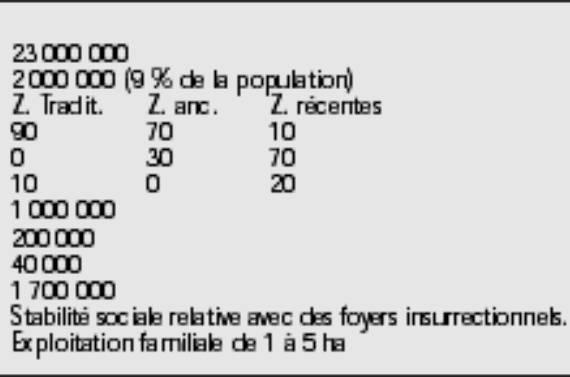 & 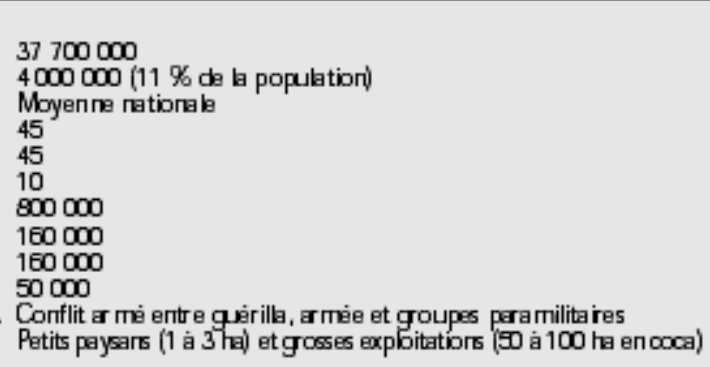 \\
\hline 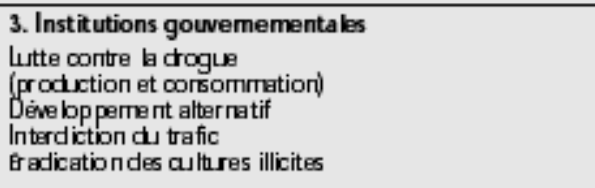 & 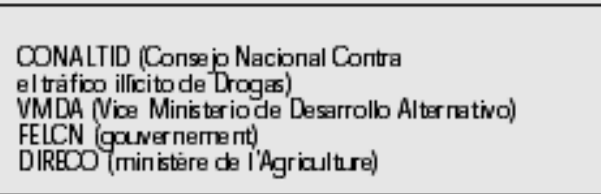 & 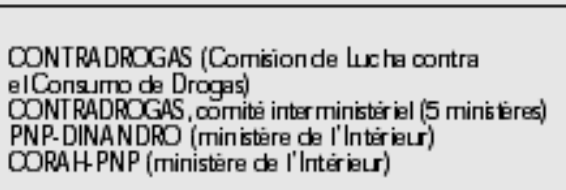 & 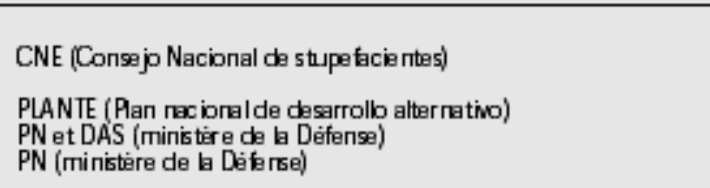 \\
\hline $\begin{array}{l}\text { 4. Politique sur les drogues } \\
\text { Interdiction } \\
\text { Eradicationdes cultures }\end{array}$ & $\begin{array}{l}\text { Par les traficants et les paysers du Chapare } \\
\text { Dars la Chapare : immédiete par las aultures récentes, }\end{array}$ & \multirow{3}{*}{ 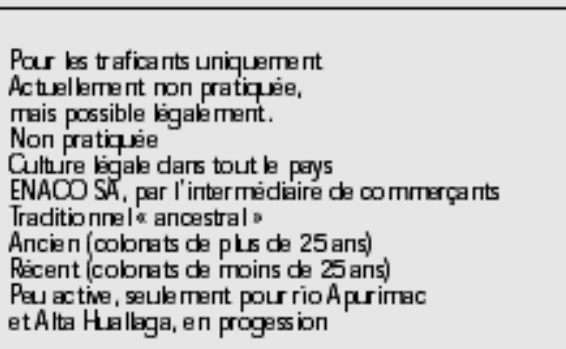 } & $\begin{array}{l}\text { Par les producters et les traficants, sur tout la pays } \\
\text { Eradication forcie }\end{array}$ \\
\hline 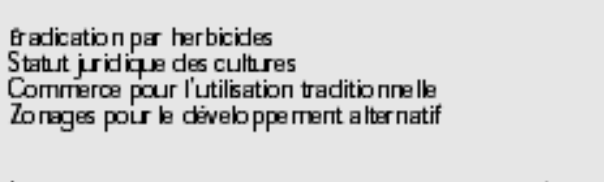 & 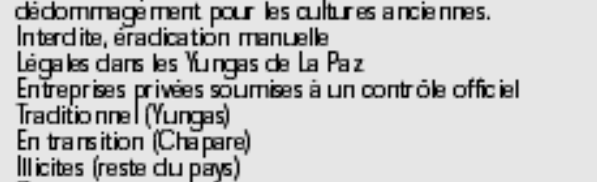 & & $\begin{array}{l}\text { Lègale et pratiqué par voie aérienne (Glyphosate et Irra zapir) } \\
\text { Culture illagale } \\
\text { lllègal }\end{array}$ \\
\hline Intervention privée dars la dèveloppement alternatif & Chapare : lièe à b Dopération inter nationale & & Peu active (gande irsécurité) \\
\hline \multicolumn{2}{|l|}{ 5. Importance éco no mique de la coca } & \multirow{5}{*}{ 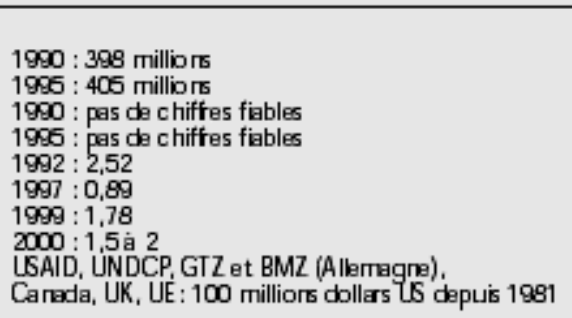 } & \\
\hline & 1996 : 350 milliors & & $\begin{array}{l}1980: 5050 \text { millibrs (2) } \\
1996: 3939 \text { milliors }\end{array}$ \\
\hline Valeur des exportatiors (estimation) & $1990: 222$ milliors & & $1990: 1112$ milliors \\
\hline $\begin{array}{l}\text { Prix moyen de la feuille de coca payé au producteur } \\
\text { (\$/kg de fauilles séchess) }\end{array}$ & $\begin{array}{l}1996: 271 \text { milliors } \\
1992: 0,90 \\
1997: 1 \\
2000: 4 \text { à } 5\end{array}$ & & $\begin{array}{l}1996 \text { : } 817 \text { milliors } \\
\text { Baisse forte de 1995 a 1999, actuellement pr been augmentation. }\end{array}$ \\
\hline Cocpèration inter ratibrale par la dèveloppernent alerratif & $\begin{array}{l}\text { LSAID, UNDCP, GTZ (Allarragne), } \\
\text { UE : } 250 \text { milliors dollas LS depuis } 1987\end{array}$ & & $\begin{array}{l}\text { BID (creidit de } 90 \text { milliors } \\
\text { dollas LS), UNDCP, GRZ: } 100 \text { milliors dollas LSdapuis } 1990\end{array}$ \\
\hline Besoins de firrancernent & $\begin{array}{l}\text { Plan Dignidad 1998-2002: } \\
\text { C60 milliors dollas LS (dors) }\end{array}$ & $\begin{array}{l}\text { Pan } 1999 \text { 2003 : dollas LS } 1000 \text { millbrs } \\
\text { (270 dugroupe consultatifi, } 500 \text { sous for me de crédit). }\end{array}$ & $\begin{array}{l}\text { PLANTE } 1998-2003: 1000 \text { milliors dollas LS } \\
\text { (700 saus forme de crédit). }\end{array}$ \\
\hline
\end{tabular}

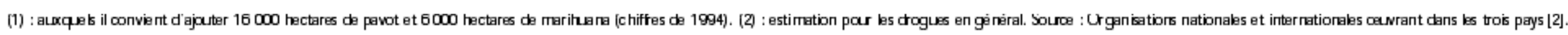

